\title{
A polinização por abelhas sob a perspectiva da Abordagem de Serviços Ecossistêmicos (ASE)
}

O presente trabalho teve por objetivo analisar os benefícios da polinização por abelhas sob o olhar da Abordagem de Serviços Ecossistêmicos (ASE). $\mathrm{O}$ artigo contou com uma revisão teórica tradicional relacionada à ASE e à Economia Ecológica e com uma revisão sistemática das publicações indexadas à plataforma Web of Science, que fizeram algum tipo de avaliação da polinização por abelhas, silvestres ou manejadas. Esta revisão ocorreu por meio de quatro etapas: busca dos artigos, triagem, extração das informações e síntese dos resultados. Durante a primeira etapa foram identificadas 182 publicações até setembro de 2020. Após etapas posteriores e leitura integral dos trabalhos, foram selecionados 45 que responderam ao problema de pesquisa aqui proposto. Os resultados desta revisão indicam um aumento expressivo de trabalhos publicados nas duas últimas décadas, sobretudo nos últimos dez anos ( $86,6 \%$ ). Dos pesquisa aqui proposto. Os resultados desta revisão indicam um aumento expressivo de trabalhos publicados nas duas últimas décadas, sobretudo nos últimos dez anos (86,6\%). Dos
benefícios identificados, $84,44 \%$ dos trabalhos associaram a polinização à produtividade rural agrícola, enquanto $8,88 \%$ avaliaram a importância deste serviço ecossistêmico para a reprodução de plantas silvestres e $6,66 \%$ à agricultura urbana. Sementes por fruto é a medida mais direta deste serviço ecossistêmico, estando diretamente ligada às métricas de tamanho e de qualidade dos frutos. Dos 45 trabalhos analisados, somente 07 trabalhos realizaram a valoração econômica e os métodos utilizados foram: Taxa de Dependência de Polinização (TDP), Custo de Reposição (CR), Análise de Rendimento Líquido (ARL) e Disposição a Pagar (DAP). Constatou-se, de forma geral, que a polinização por abelhas é amplamente reconhecida pela sua importância para algumas culturas agrícolas e para a formação de preços na agricultura. Não obstante, a ASE propõe uma análise integradora das perspectivas econômicas, ambientais e sociais que orienta a identificação de valores para além do mercado dos serviços ecossistêmicos. No caso da polinização, deve-se reconhecer também a sua contribuição para a reprodução de vegetação nativa, manutenção de paisagens e, consequentemente, para a preservação da biodiversidade.

Palavras-chave: Polinização por Abelhas; Abordagem de Serviços Ecossistêmicos; Revisão Sistemática.

\section{Bee pollination from the perspective of the Ecosystems Services Approach (ESA)}

\begin{abstract}
This study aimed to analyze the benefits of bee pollination from the perspective of the Ecosystems Services Approach (ESA). The article included a traditional theoretical review related to ASE and Ecological Economics and a systematic review of publications indexed to the Web of Science platform, that had done some kind of evaluation of pollination by bees, wild or managed. This review took place through four phases: search for articles, screening, extraction of information and synthesis of results. During the first phase, 182 publications were identified until September 2020. After subsequent stages and full reading of the works, there were selected 45 that responded to the problematic proposed in this research. The results indicated an September 2020. After subsequent stages and full reading of the works, there were selected 45 that responded to the problematic proposed in this research. The results indicated an
expressive increase of studies published in the last two decades, overall, on the last ten years $(86,6 \%)$. Of the identified benefits, $84,4 \%$ of the studies associated the pollination to the rural expressive increase of studies published in the last two decades, overall, on the last ten years ( $86,6 \%)$. Of the identified benefits, $84,4 \%$ of the studies associated the pollination to the rural
agricultural productivity, meanwhile $8,88 \%$ of the studies evaluated the significance of this ecosystems service to the wild plant's reproduction and $6,66 \%$ to the urban agriculture. Seeds by agricultural productivity, meanwhile $8,88 \%$ of the studies evaluated the significance of this ecosystems service to the wild plant's reproduction and $6,66 \%$ to the urban agriculture. Seeds by
fruit is the most direct measurement of this ecosystem service, being directly connected to the metrics of fruit size and quality. From the 45 analyzed studies, only 07 performed the economic valuation and the methods used were: Dependency Ratio (DR), Replacement Costs (CR), Yield Analysis (YA) and Willingness to Pay (WTP). It was found, in general, that bee pollination is widely recognized for its importance for agricultural crops and for the formation of prices in agriculture. Nevertheless, the ESA proposes an integrative analysis of economic, environmental and social perspectives that guides the identification of values beyond the market for ecosystem services. In the case of pollination, its contribution to the reproduction of native vegetation, maintenance of landscapes and, consequently, to the preservation of biodiversity must also be recognized.
\end{abstract}

Keywords: Bee Pollination; Ecosystems Services Approach; Systematic Review.

Topic: Desenvolvimento, Sustentabilidade e Meio Ambiente

Reviewed anonymously in the process of blind peer

Fernanda Rodrigues Vieira

Universidade Federal de Goiás, Brasil

http://lattes.cnpq.br/6204481211277898

http://orcid.org/0000-0001-9905-0371

fernanda.vy@gmail.com

Daniel Caixeta Andrade (it)

Universidade Federal de Uberlândia, Brasil http://lattes.cnpq.br/4632609286866341 http://orcid.org/0000-0002-5045-1913

daniel.andrade@ufu.br

Francis Lee Ribeiro (iD

Universidade Federal de Goiás, Brasil http://lattes.cnpq.br/1763392088652134

http://orcid.org/0000-0001-9329-2505

francisleerib@gmail.com

d

DOI: 10.6008/CBPC2179-6858.2021.004.0042
Received: 01/04/2021

Approved: 28/04/2021
Referencing this:

VIEIRA, F. R.; ANDRADE, D. C.; RIBEIRO, F. L.. A polinização por abelhas sob a perspectiva da Abordagem de Serviços Ecossistêmicos (ASE). Revista Ibero Americana de Ciências Ambientais, v.12, n.4, p.544560, 2021. DOI: http://doi.org/10.6008/CBPC21796858.2021 .004 .0042 


\section{INTRODUÇÃO}

Os serviços ecossistêmicos são essenciais para o desenvolvimento da vida no planeta, assim como para a realização das atividades econômicas e culturais da sociedade. O bem-estar humano está relacionado aos benefícios diretos e indiretos obtidos a partir da estrutura e da interação dos componentes do meio ambiente (COSTANZA et al., 1997). Podemos assim citar a provisão de água, de madeira, de oxigênio, a regulação do clima e dos nutrientes do solo, e a polinização como alguns desses serviços.

A polinização é amplamente conhecida pela sua importância para a reprodução de espécies vegetais sendo que os seus agentes, principalmente as abelhas, contribuem de forma significativa para a obtenção de diversas culturas agrícolas e para a conservação da diversidade genética da flora (GARIBALDI et al., 2016). Um terço da produção agrícola depende dos serviços ecossistêmicos de polinização animal. Um total de $94 \%$ das plantas nas zonas tropicais são polinizadas por animais, sendo que as abelhas representam cerca de $75 \%$ desse número (ELIAS et al., 2017; KLATT et al., 2013).

Estudos publicados nos últimos anos, em todo mundo, têm chamado a atenção para o declínio de populações de abelhas silvestres, principalmente, devido à intensificação das atividades agropecuárias (MWEBAZE et al., 2018; SÍLVIA et al., 2016). O aumento da demanda por polinizadores naturais não tem acompanhado a sua oferta por causa da dinâmica da globalização e do aumento populacional, o que exige uma nova configuração da agricultura (AIZEN et al., 2009; NICHOLLS et al., 2020). A expansão das áreas urbanas, das áreas para plantio e o uso excessivo de agrotóxicos têm diminuído a diversidade e a disponibilidade das abelhas, uma vez que comprometem a qualidade do seu habitat e reduz consideravelmente os recursos florais que dão suporte à vida e à reprodução desses agentes (Ś́LVIA et al., 2016).

A Abordagem de Serviços Ecossistêmicos (ASE) chama atenção para a necessidade de se reconhecer os benefícios provenientes da natureza, enfatizando a necessidade de mudança do paradigma vigente de organização socioeconômica, visando estabelecer uma nova relação entre homem e natureza. Parte-se da premissa de que a preservação do capital natural é essencial para a prosperidade da espécie humana e para o seu bem-estar e que o modelo civilizatório possui uma lógica intrínseca de destruição da base natural que suporta todas as relações sociais e econômicas estabelecidas (MARQUES, 2020).

Reconhecendo-se, pois, os riscos trazidos pelas atividades antrópicas para os serviços de polinização, é pertinente investigar como estes benefícios têm sido avaliados nas últimas décadas pela literatura especializada. A hipótese que guia este trabalho é a de que os benefícios dos serviços de polinização por abelhas são avaliados a partir da sua contribuição para a produtividade rural agrícola ou conforme valores de mercado das safras ou colheitas, deixando de refletir a complexidade dos processos ecológicos envolvidos em um serviço de regulação e a sua contribuição para a preservação da biodiversidade. Em sendo assim, nosso objetivo é analisar a polinização por abelhas por meio de uma breve revisão sistemática dos trabalhos que fizeram a avaliação dos benefícios deste serviço ecossistêmico. Considera-se que esta revisão permitirá elucidar os principais vieses nos estudos sobre o tema proposto, evidenciando se há ou não preferência pela 
dimensão econômica em detrimento de outras dimensões importantes no processo de avaliação de serviços ecossistêmicos em geral (dimensão ecológica e social).

O presente estudo está estruturado a partir desta Introdução, com uma seção posterior para a Abordagem dos Serviços Ecossistêmicos (ASE) e outra para o Serviço Ecossistêmico de Polinização, seguidas da Metodologia, Resultados e Discussão da Revisão Sistemática, Valoração Econômica da Polinização por Abelhas e Considerações Finais, o que abrange as limitações do trabalho e indicações para futuras pesquisas.

\section{REVISÃO TEÓRICA}

\section{Abordagem de Serviços Ecossistêmicos (ASE)}

Os serviços ecossistêmicos representam a interface do capital natural com o homem, sendo, portanto, as características, o arranjo de funções e de processos ecológicos que contribuem para o bem-estar humano (ANDRADE, 2015; COSTANZA, 2020). A qualidade da vida da população, assim como o seu sistema econômico, depende dos benefícios diretos e indiretos obtidos a partir da estrutura e da combinação dos componentes do meio ambiente. Os ecossistemas abrangem funções complexas e de interação entre seres vivos e não vivos, em seus habitats físicos ou biológicos, com padrões de fluxos de nutrientes que determinam a diversidade da oferta dos serviços ecossistêmicos (ANDRADE et al., 2013; CONSTANZA et al., 1991; DAHAL et al., 2018). Estes podem ser resultados de uma ou mais funções ecossistêmicas (processos ecológicos que existem independentemente dos benefícios que geram para os seres humanos) e uma função ecossistêmica pode gerar um ou mais serviços ecossistêmicos (ANDRADE, 2015).

A Avaliação Ecossistêmica do Milênio (MEA, 2005) considera quatro categorias de serviços ecossistêmicos: 1) serviços de regulação - aqueles que mantém as características de equilíbrio dos processos ecossistêmicos, como regulação climática, controle de erosão, qualidade e pureza do ar e da água; 2) serviços de suporte - relacionados à estrutura e componentes necessários para a existência dos demais serviços ecossistêmicos e a sua relação com o homem geralmente acontece de forma indireta, como por exemplo a ciclagem de nutrientes na atmosfera; 3) serviços de provisão - contemplam os produtos extraídos diretamente do meio ambiente, como por exemplo alimentos, madeira, água, recursos medicinais e ornamentais; 4) serviços de informação ou culturais - englobam os valores religiosos, espirituais, a diversidade de saberes e de etnias, as experiências e o conhecimento das comunidades, os valores estéticos e de recreação (MEA, 2005).

O termo "serviços da natureza" apareceu pela primeira vez na literatura acadêmica em 1977, em um artigo publicado na Science por Walter Westman, intitulado "How Much Are Nature's Services Worth?" (COSTANZA et al., 2017). Já a expressão "serviços ecossistêmicos" propriamente dita foi utilizada por Ehrlich et al. (1981) e Ehrlich et al. (1983) ao discorrerem sobre as causas e consequências da extinção de espécies para os ecossistemas (COSTANZA et al., 2017; EHRLICH et al., 2013; GÓMEZ-BAGGETHUN et al., 2010). Esse conceito partiu da necessidade de um enquadramento dos benefícios provenientes dos ecossistemas como "serviços" a fim de despertar o interesse público pela conservação da biodiversidade (BRAAT et al., 2012). 
Tal ideia permitiu estabelecer uma ponte entre as ciências econômicas/sociais e a ecologia, evoluindo, posteriormente, como parte do arcabouço teórico da "Economia Ecológica" que surge como uma disciplina integradora, no final da década de 1980 (CONSTANZA et al., 1991)1.

Como consequência, houve uma proliferação de conceitos, metodologias e estudos de caso envolvendo as relações entre sistemas econômicos e ecológicos e uma grande concentração de métodos para estimar o valor econômico dos serviços ecossistêmicos (BRAAT et al., 2012; COSTANZA et al., 2017). Um grande marco foi o trabalho de Costanza et al. (1997) que realizou a valoração de dezessete serviços ecossistêmicos, em dezesseis biomas em todo globo terrestre, estimando um valor médio de US\$33 trilhões por ano. Não obstante às críticas posteriores que argumentavam se tratar de um valor subestimado devido aos atributos intangíveis dos bens ambientais, a ideia era justamente demonstrar que a conservação dos ecossistemas poderia ser mais valiosa do que a sua exploração (COSTANZA et al., 2017).

O conceito de serviços ecossistêmicos ultrapassou as fronteiras acadêmicas e passou a integrar também as ferramentas de políticas públicas, programas de empresas privadas e de organizações sem fins lucrativos (BAGGETHUN et al., 2010). O debate entrou para agenda política internacional no início da década de 2000 com a Avaliação Ecossistêmica do Milênio (AEM), encomendada pela Organização das Nações Unidas - ONU (BAGGETHUN et al., 2010; MEA, 2005). A AEM, por meio do resultado de estudos técnicos e científicos, teve um papel fundamental para a disseminação da importância da conservação e do uso sustentável dos ecossistemas e sua relação com o bem-estar da sociedade. Nessa ocasião foram analisadas as mudanças nos fluxos e na oferta dos serviços ecossistêmicos e a sua relação com a qualidade de vida humana, por meio de diagnósticos e projeções de cenários elaborados com base nas ações antrópicas e de devastação do capital natural (MEA, 2005).

A Abordagem de Serviços Ecossistêmicos (ASE) pode, pois, ser considerada como uma perspectiva teórico-metodológica que visa estudar fenômenos socioeconômicos a partir de sua releitura em termos biofísicos, explicitando a origem, a dependência e as conexões existentes entre o bem-estar humano e os serviços da natureza. Trata-se, em última instância, de uma abordagem que objetiva reduzir o reducionismo teórico e metodológico típicos de uma análise puramente economicista, conferindo, assim, maior pluralismo e holismo às investigações sobre as interfaces entre ciências sociais e ciências naturais. Por sua natureza transdisciplinar, ancora-se fortemente na Economia Ecológica e enfatiza a importância do capital natural e seus fluxos intangíveis ${ }^{2}$ (ANDRADE, 2015).

A ASE, assim, reconhece a existência de quatro categorias de capital que contribuem para o bemestar humano: o capital humano, representado pelo estoque de conhecimento acumulado pela espécie humana; o capital social, representado pelas interações sociais e instituições; o capital construído, representado pela infraestrutura física criada pelas sociedades; e o capital natural, que engloba todo o

\footnotetext{
${ }^{1}$ De maneira genérica, a Economia Ecológica é uma proposta de estudo transdisciplinar com o objetivo de compreender as interfaces entre sistemas socioeconômicos e sistemas naturais. Enfatiza a dependência visceral das atividades econômicas em relação aos serviços da natureza, assim como a importância dos serviços ecossistêmicos como categoria analítica fundamental. Ver Baggethun et al. (2010) para uma análise aprofundada sobre o uso dos serviços ecossistêmicos na teorização e prática da ciência econômica.

${ }^{2}$ Dentre estes fluxos intangíveis, destacam-se os serviços de regulação, categoria na qual estão os serviços de polinização, objetivo deste artigo.
} 
estoque de recursos naturais disponíveis, juntamente com os fluxos de serviços gerados por meio das interações entre biodiversidade e os fatores abióticos (temperatura, solos, etc.). Nesse sentido, A ASE procura compreender de que forma estes quatro tipos de capital devem interagir entre si de maneira a produzir o almejado desenvolvimento sustentável (COSTANZA, 2020).

A partir daí, a construção de um novo paradigma centrado na relação homem e natureza demanda uma reavaliação do significado convencional de bem-estar que considera a percepção do indivíduo para além da afluência material. Esta deve ser ampliada, sob uma perspectiva sistêmica, que tem por objetivo considerar o bem-estar de todos os ecossistemas que sustentam a vida e não só a dimensão humana (GOULD et al., 2020).

\section{O serviço ecossistêmico de polinização}

A polinização é considerada um serviço ecossistêmico de regulação, imprescindível para a reprodução de espécies vegetais. Trata-se do transporte dos grãos de pólen contidos nas anteras de uma flor para o seu estigma ou para o estigma de outra planta (COSTA et al., 2013). Esta acontece de forma cruzada, quando o pólen de uma flor é transferido para outra flor da mesma espécie em diferentes plantas, ou de forma direta (autopolinização), quando a transferência do pólen da antera para o estigma acontece em uma mesma flor ou em outra flor, na mesma planta (NASCIMENTO et al., 2012). Tal mecanismo pode ser realizado por agentes bióticos ou abióticos (VIEIRA et al., 2010). Em relação às espécies que possuem flores, $87,5 \%$ da polinização é feita por algum tipo de animal. Esses agentes polinizadores podem ser vertebrados, como por exemplo, morcegos, aves, répteis, e invertebrados como abelhas, moscas, borboletas e besouros (NASCIMENTO et al., 2012).

Em todo mundo existem cerca de 20.000 espécies de abelhas (FONSECA et al., 2010) e o que há de comum para toda essa biodiversidade é a dependência das flores para a sua alimentação, uma vez que o pólen oferece a proteína e o néctar, os açúcares. Estudos recentes indicam o declínio do número de agentes polinizadores e, consequentemente, dos serviços de polinização por abelhas silvestres e domesticadas em todo o mundo (MWEBAZE et al., 2018; SÍLVIA et al., 2016). Evidências em várias regiões do globo, como a queda do número de colônias de abelhas nos Estados Unidos, redução da diversidade de espécies de abelhas selvagens na Europa, estão associadas ao desmatamento que provoca a perda e a fragmentação de habitats. Outra questão que tem chamado a atenção é que a quantidade de polinizadores naturais não tem acompanhado a expansão das culturas agrícolas que dependem dos serviços de polinização (AIZEN et al., 2009).

As abelhas percorrem cerca de $90 \%$ das culturas agrícolas, enquanto as moscas visitam $30 \%$ e os vertebrados $6 \%$ (OLLERTON et al., 2011). Os serviços de polinização por abelhas são fundamentais para o processo de produção de alimentos em todo o mundo, ainda que grande parte das espécies sejam capazes de realizar a polinização direta. Como é o caso do cultivo do tomate, não obstante a ocorrência de autopolinização, quando está é realizada por abelhas silvestres há uma elevação da qualidade e da produtividade dos frutos (aumento de peso e de tamanho), além de possibilitar também a uniformização do 
seu amadurecimento (GAGLIANONE et al., 2015; NASCIMENTO et al., 2012). Os serviços de polinização em áreas agrícolas têm ocorrido tanto por meio de abelhas silvestres quanto como o auxílio de espécies domesticadas, como por exemplo, as abelhas Apis melifera L. Estas são utilizadas devido à facilidade em seu manejo, sua disponibilidade e adaptação aos diferentes ecossistemas (GARIBALDI et al., 2016; SÍLVIA et al., 2016).

Os serviços ecossistêmicos de polinização foram valorados pela primeira vez por Costanza et al. (1997), estimados em US\$112 bilhões ao ano. Gallai (2008) utilizando dados da agricultura mundial de 2005 obteve um valor de $€ 153$ bilhões para o mesmo serviço. No Brasil, Giannini et al. (2015b) revisaram a dependência das culturas por polinizadores e obtiveram um valor econômico de US\$12 bilhões, equivalente a $30 \%$ da renda agrícola anual do país. Metade desse valor equivale à polinização da soja, o que ainda é considerado controverso porque a cultura da soja apresenta uma dependência moderada de polinizadores. Não obstante, a valoração desses serviços é fundamental para auxiliar o poder público em suas tomadas de decisão, assim como para orientar os agentes econômicos no ajuste do nível ótimo de produção que leve em conta os custos socioambientais de suas atividades. Todavia, deve-se mencionar que a valoração é apenas uma ferramenta para melhor gestão do capital natural e seus serviços, não podendo ser considerada uma panaceia dentro do objetivo maior de conservação ambiental (ANDRADE et al., 2013).

Também vale ressaltar que os benefícios da polinização por abelhas, embora seja mais reconhecido pela sua ligação com a produção de alimentos, é fundamental para a reprodução de espécies nativas e para o equilíbrio dos ecossistemas, como será investigado pela revisão sistemática das próximas seções.

\section{METODOLOGIA}

Trata-se aqui de uma pesquisa de natureza teórica que buscou identificar quais são os principais benefícios provenientes da polinização por abelhas e de que forma estes têm sido avaliados pela literatura especializada, que os classifica como serviços ecossistêmicos.

A pesquisa foi desenvolvida em duas fases. Para o cumprimento da primeira fase foi realizado um levantamento bibliográfico tradicional sobre serviços ecossistêmicos e os últimos avanços nesse debate sob a ótica da Economia Ecológica. Foram pesquisados os artigos mais recentes de autores insignes que tratam do tema nas plataformas Scielo, Web of Science e Scopus. Com isso foi possível compilar as principais tendências e vieses que serviram de orientação para a discussão dos resultados da etapa posterior.

Durante a segunda fase da pesquisa foi feita uma revisão sistemática na plataforma Web Science com o propósito de verificar todas as publicações que se propuseram a avaliar os benefícios dos serviços de polinização por abelhas. O cumprimento desta fase se deu de acordo com as orientações do Preferred Reporting Items for Systematic Reviews and Meta-Analyses, The PRISMA Statement (MOHER et al., 2009), cumprindo as seguintes etapas: busca dos artigos, triagem, extração das informações e a síntese dos resultados, Figura 1.

A busca dos trabalhos, etapa 1, ocorreu por meio da seguinte equação: TS=(ecosystem services AND bee pollination) AND TS= (valuation OR measure) na plataforma de busca avançada da Web of Science. 
Foram levantadas 182 publicações até setembro de 2020. Na etapa 2, de triagem, foram lidos os resumos e selecionadas as publicações que tratavam especificamente da polinização por abelhas ( $N=67)$. Ao se realizar uma leitura completa dos trabalhos durante a etapa 3 , foram eleitos somente os trabalhos que de alguma forma respondiam à pergunta de pesquisa e que fizeram algum tipo de avaliação deste serviço ecossistêmico $(\mathrm{N}=45)$. Posteriormente, os dados bibliométricos e dos tipos de benefícios avaliados foram extraídos, sintetizados e discutidos à luz da abordagem de serviços ecossistêmicos (ASE).
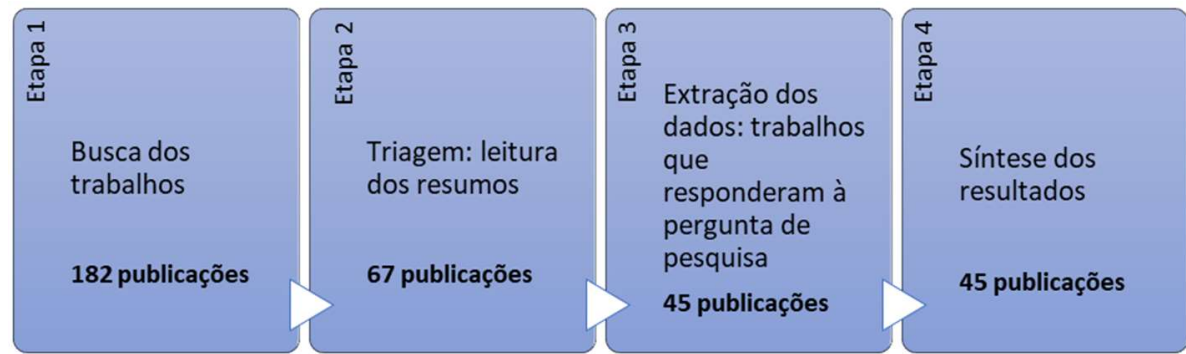

Figura 1: Etapas da revisão sistemática realizada conforme orientação do Preferred Reporting Items for Systematic Reviews and Meta-Analyses. Fonte: Moher et al. (2009).

Dos trabalhos analisados $(\mathrm{N}=45)$, somente 07 realizaram a valoração econômica dos serviços de polinização por abelhas. Os métodos identificados foram classificados em: Taxa Dependência de Polinização (TDP), Custo de Reposição (CR), Análise de Rendimento Líquido (ARL) e Disposição a Pagar (DAP).

\section{RESULTADOS E DISCUSSÃO}

A busca na plataforma Web of Science identificou 182 publicações com a nossa equação de pesquisa avançada. Após a verificação dos resumos e das palavras-chave foram selecionados 67 trabalhos para que fosse feita uma leitura integral, dos quais somente 45 responderam à pergunta de pesquisa proposta por este trabalho. Grande parte dos trabalhos descartados tratavam da polinização por animais ou por insetos de forma generalizada e não só de abelhas, conforme o objetivo desta revisão. Foram excluídas também as publicações que investigaram exclusivamente aspectos de entomologia e taxonomia das abelhas. Para a elegibilidade dos 45 trabalhos selecionados, seguiu-se a definição de serviços ecossistêmicos como sendo os benefícios diretos e indiretos decorrentes do capital da natureza. Assim, as publicações escolhidas realizaram a identificação e avaliação de pelo menos um dos benefícios provenientes da polinização por abelhas, selvagens ou manejadas, sob um viés predominantemente ecológico $(\mathrm{N}=35)$ ou econômico $(\mathrm{N}=10)$.

A busca realizada não colocou nenhuma restrição de tempo para o levantamento das pesquisas. Não obstante, o período dos trabalhos selecionados se inicia em 2001 e vai até o fim de 2019, com picos de publicação nos anos de 2014, 2015 e 2019 (Figura 2a). As revistas que apresentaram o maior número de publicações foram: Agriculture, Ecosystems and Environment $(\mathrm{N}=8)$, Journal of Applied Ecology $(\mathrm{N}=5)$ e Journal of Economic Entomology ( $\mathrm{N}=4)$, Figura 2b.

Os resultados da revisão sistemática sobre os benefícios da polinização por abelhas indicam um aumento expressivo de trabalhos publicados nas duas últimas décadas, sobretudo nos últimos dez anos $(86,6 \%)$. A ameaça de uma possível crise de polinização devido às consequências do aumento da demanda 
mundial por alimentos e o comprometimento dos habitats destes insetos, em decorrência das ações antrópicas, tem atraído a atenção da comunidade científica, política e população em geral (AIZEN et al., 2009; SÍLVIA et al., 2016). Como consequência, a polinização está sendo reconhecida cada vez mais como um serviço ecossistêmico de regulação fundamental para a produção de alimentos e para a preservação da biodiversidade (BPBES, 2019).
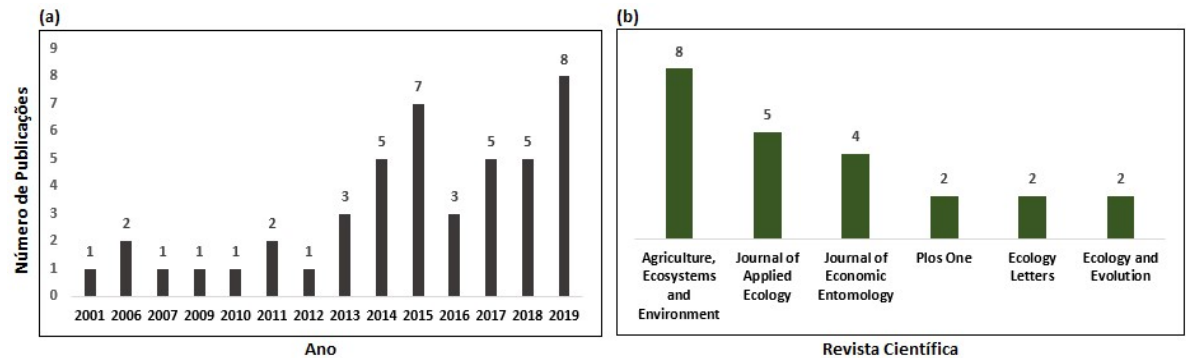

Figura 2: Publicações que realizaram alguma forma de avaliação dos benefícios dos serviços de polinização por abelhas, tanto sob um viés econômico quanto ecológico. (a) Distribuição anual das 45 publicações selecionadas que avaliaram a polinização por abelhas de 2001 até 2019. (b) Número de publicações por revista científica que foram selecionadas a partir da busca na plataforma Web of Science.

Das 45 publicações selecionadas, 28,88\% apresentaram algum tipo de avaliação dos benefícios dos serviços de polinização por abelhas como objetivo principal do trabalho, enquanto $71,12 \%$ realizaram essas avaliações como parte de outras propostas. Desses 45 trabalhos, $22,22 \%$ tinham a economia como área principal de estudo, sendo que somente $15,55 \%(N=07)$ realizaram a valoração econômica da polinização. Os trabalhos com o maior número de citações podem ser verificados na Tabela 1.

Tabela 1: Trabalhos selecionados por esta revisão que foram citados um maior número de vezes, de acordo com a análise realizada pela plataforma Web of Science.

\begin{tabular}{|c|c|c|c|c|}
\hline publicação & periódico & & $\begin{array}{l}\text { número de vezes em que o artigo foi } \\
\text { citado }\end{array}$ & culturas avaliadas \\
\hline Winfree et al. (2007) & Ecology Letters & & 280 & melancia \\
\hline Breeze et al. (2011) & $\begin{array}{l}\text { Agriculture, } \quad \text { Ecosystems } \\
\text { Environment }\end{array}$ & and & 153 & múltiplas culturas \\
\hline Winfree et al. (2011) & Ecological Economics & & 90 & melancia \\
\hline Martins et al. (2015) & $\begin{array}{l}\text { Agriculture, } \\
\text { Environment }\end{array}$ & and & 85 & maçã \\
\hline Blanche et al. (2006) & Journal of Applied Ecology & & 81 & macadâmia \\
\hline Isaacs et al. (2010) & Journal of Applied Ecology & & 72 & mirtilo \\
\hline Woodcock et al. (2013) & $\begin{array}{l}\text { Agriculture, Ecosystems } \\
\text { Environment }\end{array}$ & and & 70 & colza \\
\hline Mallinger et al. (2015) & Journal of Applied Ecology & & 68 & maçã \\
\hline Cariveau et al. (2013) & Ecology Letters & & 60 & $\begin{array}{l}\text { mirtilo, cereja } \\
\text { melão }\end{array}$ \\
\hline Giannini et al. (2015a) & Apidologie & & 49 & múltiplas culturas \\
\hline
\end{tabular}

A grande maioria dos autores das publicações analisadas está filiada a instituições localizadas em países considerados desenvolvidos $(N=41)$, enquanto um número reduzido $(N=4)$ encontra-se em países em desenvolvimento (Figura 3). Os países de maior expressividade onde se localizam as instituições dos autores correspondentes, como ilustra a Figura 3a, são: Estados Unidos ( $N=21)$, Reino Unido $(N=6)$, Austrália $(N=2)$, Brasil ( $N=2)$, China ( $N=2$ ) e Espanha ( $N=2)$. Da mesma forma, em relação aos locais em que foram conduzidas as pesquisas (Figura $3 b$ ), o maior número também se encontra em países desenvolvidos $(\mathrm{N}=38)$ em contraposição aos países em desenvolvimento $(\mathrm{N}=7)$, sendo os principais: Estados Unidos $(\mathrm{N}=21)$, Reino 
Unido ( $\mathrm{N}=4)$, China $(\mathrm{N}=4)$ e Brasil $(\mathrm{N}=4)$.

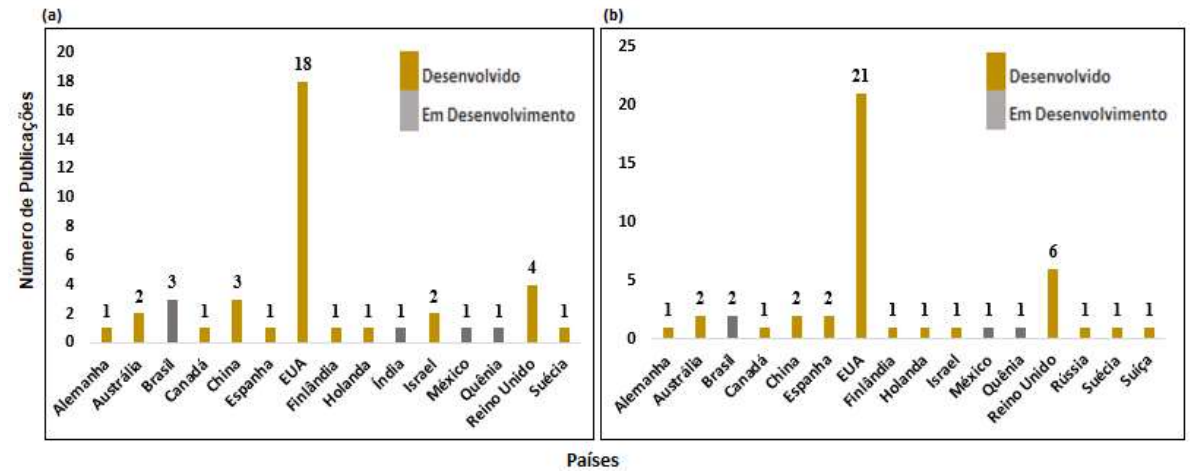

Figura 3: Distribuição das publicações em relação aos países de filiação e de realização dos trabalhos selecionados por esta revisão sistemática. (a) Número de publicações por países em que as instituições dos autores principais estavam

filiadas. (b) Número de publicações por países onde foram conduzidas as pesquisas, em que foram excluídas as publicações de natureza teórica ( $\mathrm{N}=4)$.

Compreender e gerenciar o serviço de polinização tem sido um processo difícil que vem se esbarrando em impedimentos taxonômicos e escassez de dados, especialmente nos trópicos (LIU et al., 2017). Há uma expressiva deficiência de dados sobre culturas comerciais, regionalmente importantes, e que são essenciais para a segurança alimentar de milhares de pessoas, especialmente nos países em desenvolvimento (PORTO et al., 2020). Não obstante, quase a totalidade das pesquisas analisadas aqui $(84,4 \%)$ foram conduzidas em países considerados desenvolvidos. No caso da polinização por abelhas, especificamente, é importante que se faça a identificação das espécies para que se entenda a real contribuição destas para as culturas avaliadas. Geralmente, as pesquisas que fazem análise taxonômica e a associa aos benefícios de polinização necessitam de equipes interdisciplinares e de um período, que muitas vezes, ultrapassa um ano para a realização dos experimentos, demandando investimentos e condições estruturais adequadas.

Em relação à natureza dos estudos verificados, a quase totalidade foi de ordem empírica $(\mathrm{N}=41)$ enquanto os demais $(\mathrm{N}=4)$ tiveram essência teórica. Deste total, $82,22 \%$ realizaram a identificação das espécies de abelhas com o auxílio de profissionais de taxonomia ou de registros de trabalhos pré-existentes. Em relação ao manejo dessas abelhas, $40 \%$ estudaram tanto as espécies manejadas quanto as selvagens, $51,11 \%$ avaliaram a polinização de abelhas selvagens, $6,66 \%$ investigaram somente as abelhas manejadas e $2,22 \%(N=1)$ não especificou a origem das abelhas.

No que tange à natureza do método as publicações apresentaram as seguintes classificações: modelagem ( $N=22)$, experimental $(N=19)$, levantamento bibliográfico $(N=3)$ e revisão sistemática $(N=1)$ (Figura 4a). Quanto à abrangência geográfica das pesquisas, os benefícios da polinização foram avaliados de forma local ( $N=31)$, regional $(N=7)$ e nacional $(N=3)$ e sem delimitação da área de estudo $(N=2)$, Figura $4 b$.

A caracterização da paisagem é fundamental para os estudos de abelhas e polinização. A modelagem permite aos pesquisadores compreender de que forma a polinização pode ser afetada por aspectos relacionados ao clima, uso do solo, fragmentação e isolamento de habitats e urbanização (BUCHHOLZ et al., 2020; YOUNG et al., 2018). O suprimento de polinização, por exemplo, foi modelado por Davis (2017) em 
uma paisagem usando o módulo de polinização de culturas do InVEST 2.1 no ArcGIS 10.0. O modelo utilizou estimativas de especialistas sobre ninhos e recursos florais em toda a paisagem para criar um mapa do habitat de origem para polinizadores. Com base na distribuição do habitat de origem e na faixa de forrageamento dos polinizadores, o modelo calculou um índice relativo da abundância das abelhas para cada local na paisagem. Este mapa espacialmente explícito pode ser considerado um índice de oferta de polinização. Assim, imagens de satélite associadas a outros conjuntos de dados espaciais permite que os pesquisadores possam compreender o comportamento das abelhas em relação às perdas de habitat (GALBRAITH et al., 2015).

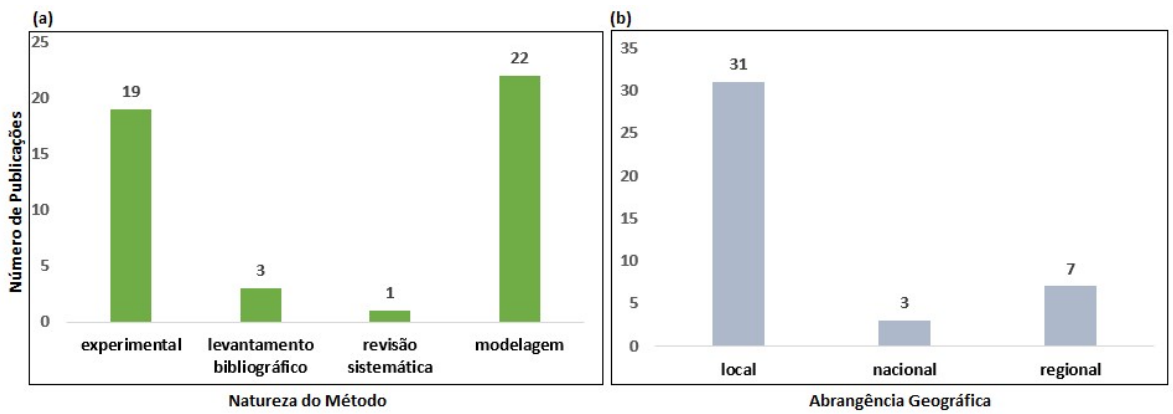

Figura 4: Natureza do método e abrangência geográfica das publicações selecionadas. (a) Número de publicações de acordo com a natureza do método utilizado. (b) Quantidade de trabalhos publicados relacionados à abrangência geográfica utilizada.

Percebeu-se a combinação de métodos de diferente natureza para uma quantidade expressiva dos trabalhos que, além da modelagem para a compreensão das variáveis ambientais que influenciam as abelhas, também realizaram técnicas experimentais para análise da polinização. Das características dos frutos medidos, sementes por fruto é a medida mais direta deste serviço ecossistêmico. A produção de sementes está ligada diretamente às métricas de tamanho e de qualidade, determinantes para a estipulação de valores de mercado de diversas frutas. Frutos de maçã, por exemplo, com um conjunto de sementes completo podem apresentar um formato maior e mais uniforme. Este processo de avaliação é obtido por meio da comparação dos frutos da polinização aberta e daqueles derivados de flores ensacadas (BRELAND et al., 2018; MALLINGER et al., 2015; ROGERS et al., 2014).

Quanto às escalas espaciais dos estudos investigados, os resultados mostram a predominância das abrangências locais $(68,8 \%)$ devido às especificidades da oferta de polinização em relação às características do ambiente e da dependência que cada cultura tem deste serviço ecossistêmico. A relação entre os serviços de polinização e a produção agrícola depende fortemente do contexto, o que às vezes dificulta a aplicação de um estudo realizado na Europa para a América do Sul (PORTO et al., 2020).

A maior parte dos trabalhos, tanto da área de economia quanto da área de ecologia identificaram os benefícios diretos da polinização como sendo aqueles relacionados à produtividade rural agrícola $(\mathrm{N}=38)$, como por exemplo aumento do número de sementes e bagas de frutos que resulta em maior qualidade e aparência. Uma pequena parte $(\mathrm{N}=04)$ associou diretamente esses benefícios à reprodução de plantas silvestres, enquanto os demais $(\mathrm{N}=03)$ os relacionaram à agricultura urbana. Dos trabalhos designados para a análise $(\mathrm{N}=45)$, a grande maioria ( $\mathrm{N}=37$ ) avaliou a polinização para apenas uma cultura, ao passo que uma 
parcela mínima $(\mathrm{N}=2)$ a fez para duas espécies e o restante $(\mathrm{N}=6)$ para múltiplas culturas ou espécies. As culturas mais investigadas foram: mirtilo $(N=6)$, maçã $(N=5)$, melancia $(N=3)$ e morango $(N=3)$.

Das publicações selecionadas, 93,33\% apontaram riscos decorrentes da mudança de uso do solo e utilização de fertilizantes e agrotóxicos como principais ameaças para a continuidade da oferta dos serviços de polinização. As consequências mencionadas foram: perda de habitats para forrageamento e nidificação (SUTTER et al., 2017), disseminação de patógenos e propagação de espécies invasoras (GALBRAITH et al., 2015), perda de diversidade filogenética (PFISTER et al., 2017), declínio da quantidade de populações de abelhas (BALZAN, 2017; MARTINS et al., 2015), urbanização (BIRKIN et al., 2015) e aumento da demanda por polinizadores (ARTZ et al., 2013).

A previsão de aumento da população mundial de 7,6 bilhões para 9,2 bilhões até 2050 provocará um aumento da demanda pelos serviços de polinização (MSU, 2018). Com o declínio das populações de abelhas silvestres e manejadas em todo o mundo e com a elevação da produção de alimentos, os custos e os preços dos alimentos tendem a subir. A agricultura é a maior beneficiária dos serviços de polinização e ao mesmo tempo a principal impulsionadora de sua diminuição. A pressão desta atividade sobre os ecossistemas exigirá a adoção de práticas menos agressivas ao meio ambiente, assim como a restauração de habitats naturais incorporados às paisagens agrícolas para o suporte dos serviços de polinização (RUNDLÖF et al., 2018).

Vale mencionar que as áreas urbanizadas tendem a aumentar à medida em que a população humana cresce e os espaços verdes se fazem cada vez mais necessários para o equilíbrio ecológico das cidades (BIRKIN et al., 2015). Existe uma estimativa de que $68 \%$ da população humana viverão em áreas urbanas até 2050 (UN, 2018), o que demandará estratégias alternativas de produção de alimentos. Assim, a agricultura urbana se apresenta como forte opção que pode proporcionar segurança alimentar e produção sustentável em pequenas escalas e que também necessitará dos serviços de polinização por abelhas (NICHOLLS et al., 2020).

\section{Valoração econômica da polinização por abelhas}

Quanto aos trabalhos que realizaram a valoração econômica dos serviços de polinização por abelhas $(\mathrm{N}=7)$, os métodos utilizados foram: Taxa de Dependência de Polinização - TDP ( $N=1)$, Custo de Reposição $C R(N=1)$, Análise de Rendimento Líquido - ARL $(N=4)$ e Disposição a Pagar - DAP $(N=1)$. Os valores encontrados são representados na Tabela 2 para diferentes escalas temporais e espaciais, assim como para determinadas ou múltiplas culturas.

Os métodos de valoração medem diferentes aspectos dos benefícios dos serviços de polinização e podem ser apropriados para diferentes escalas (MWEBAZE et al., 2018). Em nossa análise, apenas dois artigos avaliaram economicamente a polinização por abelhas em escala nacional. Para o Reino Unido, Mewbaze et al. (2018) estimaram o valor de $£ 842$ milhões ao ano para as culturas dependentes deste serviço ecossistêmico e no Quênia, Kasina et al. (2009) encontraram o valor de US\$8.544.140 ao ano para quatro espécies de feijão, tomate, girassol e pimentão. Em âmbito regional, a polinização por abelhas foi estimada, anualmente, em US\$1,424 milhões para o cultivo de mirtilos na Carolina do Norte (ROGERS et al., 2014) e em US\$3,63 milhões para melancia em Nova Jersey e Pensilvânia (WINFREE et al., 2011), nos Estados Unidos. 
Nos Estados de Maine e de Massachussetts, também nos Estados Unidos, a polinização foi estimada em US\$613 e US\$689 por hectare, a cada ano, para mirtilos e cerejas (HOSHIDE et al., 2018). Já em escala local, foram obtidos valores anuais de US\$ 564.000 e US\$ 246.000 referentes às culturas dependentes de polinização nos municípios do entorno da Serra da Bocaina e Mata do Jambreiro, respectivamente, no Brasil (HIPÓLITO et al., 2019). O valor para a polinização de maracujá em pequenas propriedades rurais dos municípios de Toledo e Itariri no Estado de São Paulo - Brasil, foi de US\$ 2.583 para cada hectare, referente a cada dois anos (POPAK et al., 2019).

Tabela 2: Publicações que realizaram a valoração econômica dos serviços de polinização por abelhas.

\begin{tabular}{|c|c|c|c|c|c|c|}
\hline publicação & método & local do estudo & cultivo & valores & & $\begin{array}{l}\text { ano } \\
\text { base }\end{array}$ \\
\hline Hipólito et al. (2019) & TDP & $\begin{array}{l}\text { Municípios do entorno da Serra da } \\
\text { Bocaina - PA e Mata do Jambreiro - } \\
\text { MG }\end{array}$ & açaí & $\begin{array}{l}\text { US\$564.000 } \\
\text { US\$246.000/ano }\end{array}$ & e & 2016 \\
\hline Popak et al. (2019) & CR & $\begin{array}{l}\text { Pequenas fazendas perto de Pedro } \\
\text { Toledo e Itariri - SP }\end{array}$ & maracujá & US\$2.583.000/2 anos & & 2013 \\
\hline Hoshide et al. (2018) & ARL & Maine e Massachussetts & mirtilo e cereja & $\begin{array}{l}\text { US\$613/ha } \\
\text { US\$689/ha/ano }\end{array}$ & $\mathrm{e}$ & 2009 \\
\hline Mwebaze et al. (2018) & DAP & Reino Unido & $\begin{array}{l}\text { todas as culturas } \\
\text { dependentes }\end{array}$ & £842 milhões/ano & & 2011 \\
\hline Rogers et al. (2014) & ARL & Carolina do Norte & mirtilo & US\$1.424.000/ano & & 2011 \\
\hline Winfree et al. (2011) & ARL & Nova Jersey e Pensilvânia & melancia & US\$3.630.000/ano & & 2005 \\
\hline Kasina et al. (2009) & ARL & Quênia & $\begin{array}{l}\text { feijão, tomate, } \\
\text { pimentão e maracujá }\end{array}$ & US\$8.544.140/ano & & 2005 \\
\hline
\end{tabular}

De acordo com o conceito de valor econômico total (VET), o valor econômico dos serviços de polinização deve considerar os três principais componentes: valor de uso, valor de opção e valor de não uso. O valor de uso diz respeito aos benefícios da polinização usufruídos direta ou indiretamente, como por exemplo a produtividade de culturas agrícolas dependentes das abelhas. $\mathrm{O}$ valor de opção refere-se às possibilidades futuras de se usar o recurso da natureza e o valor de não uso está relacionado aos valores intrínsecos do serviço ecossistêmico, independentemente da sua utilização por parte dos indivíduos (MWEBAZE et al., 2018).

Conforme os resultados desta pesquisa, das publicações que realizaram a valoração econômica da polinização por abelhas, aqueles que utilizaram os métodos de Taxa de Dependência de Polinização - TDP (HIPÓLITO et al., 2019), Custo de Reposição - CR (POPAK et al., 2019) e Análise de Rendimento Líquido -ARL (HOSHIDE et al., 2018; KASINA et al., 2009; ROGERS et al., 2014; WINFREE et al., 2011) foram capazes de captar somente os valores de uso do serviço. Embora cada um destes métodos apresente especificidades para a realização dos cálculos, todos se concentraram nos benefícios para os produtores, com base no valor de mercado das culturas ou no custo da polinização alternativa ou (WINFREE et al., 2011).

A TDP, amplamente utilizada para escalas espaciais maiores diz respeito ao valor de produção de safras dependentes de polinização. Presume-se que o rendimento da produção caia quando os polinizadores diminuem. Assim, o valor da safra é multiplicado pela taxa de dependência da polinização animal apresentada por Klein (2007). Essa classificação se divide em quatro categorias: (1) essencial; a produção é reduzida em $90 \%$ ou mais quando faltam serviços de polinização; (2) alto: há uma redução de $40 \%$ a $90 \%$; (3) modesto: diminuição de 10\% a 40\%; (4) pouco: redução de 0\% a 10\% (AIZEN et al., 2009; KLEIN et al., 2007). 
O MCR está relacionado à alternativa para a substituição do serviço ecossistêmico, seja por meio de uma tecnologia ou de um organismo que exercerá o mesmo tipo de função. No caso da polinização por abelhas nativas trata-se do cálculo da quantidade e dos valores financeiros necessários para a reposição por abelhas manejadas (POPAK et al., 2019; WINFREE et al., 2011) ou polinização manual. Já a ARL analisa o aumento de produtividade da cultura agrícola na presença de polinização, considerando o lucro líquido das colheitas como alternativa mais realista para estimar o valor dos polinizadores selvagens. Ao contrário do valor da produção, a receita líquida da fazenda e a conta do lucro líquido será de acordo com a contabilização dos custos de produção associados ao excedente que for decorrente da polinização por abelhas. Deixar de incluir estes e outros custos de produção das estimativas de avaliação da polinização pode superestimar a contribuição deste serviço ecossistêmico para a produção de alimentos (HOSHIDE et al., 2018).

Para a captação dos valores de não uso ou valores intangíveis dos bens ou serviços ecossistêmicos, para os quais não existem valores de mercado, tem sido utilizado o Método de Valoração Contingente - MVC, baseado na Disposição a Pagar - DAP ou Disposição a Aceitar - DAC dos agentes econômicos. Em nossa análise, o MVC foi utilizado para estimar a DAP dos contribuintes para a elaboração de políticas públicas e programas de apoio aos serviços de polinização (MWEBAZE et al., 2018). Hoshide et al. (2018), além da receita líquida atribuível à polinização para mirtilos e cerejas, também investigaram a DAP dos produtores e consumidores para ações que visassem a proteção das abelhas. Os produtores de ambas as safras estavam dispostos a investir anualmente de US\$140 a US\$188, para cada hectare, enquanto os consumidores se dispuseram a contribuir cerca de 6,7 vezes mais para a proteção desses invertebrados.

Dessa forma, O MVC conta com a percepção do indivíduo acerca dos benefícios provenientes da natureza, o que pressupõe que isso ocorra de acordo com as informações disponíveis e preferências de cada um. Não obstante, as pessoas nem sempre sabem da complexidade e da importância dos ecossistemas para a vida no planeta e a definição de bem-estar social baseado na agregação do bem-estar individual deve ser reavaliada. $\mathrm{O}$ avanço da discussão da ASE pressupõe que uma nova concepção de bem-estar inclua, além dos indivíduos e da sociedade, a preservação do capital natural (COSTANZA, 2020).

Reconhece-se assim a importância da valoração econômica ambiental como exercício capaz de contribuir para a gestão eficiente e racional dos recursos da natureza, que pode resultar em incentivos para a preservação do capital natural (ANDRADE, 2015). Não obstante, além da eficiência alocativa, a valoração deve incorporar aspectos sociais e ecológicos em seu escopo (GOULD et al., 2020). Para além da métrica monetária, que visa captar diferentes aspectos dos valores dos serviços ecossistêmicos, existe uma complexidade de fatores ainda desconhecidos que podem colocar em risco a continuidade da oferta destes serviços. Quando o funcionamento do ecossistema não é completamente compreendido, a valoração marginal é insuficiente para direcionar mudanças ou intervenções que poderão ocasionar uma disrupção de processos que dão suporte aos serviços ecossistêmicos (FARLEY, 2008).

Para os serviços de regulação, como é o caso da polinização, esta mensuração deve considerar os impactos biofísicos que o serviço exerce sobre o ambiente local e ecossistemas interligados. Os custos ambientais, a partir daí, não podem ser dimensionados meramente pelos custos de reposição do serviço, 
como por exemplo, a polinização manual.

\section{CONCLUSÕES}

O presente trabalho teve por objetivo analisar a polinização por abelhas sob a perspectiva da Abordagem de Serviços Ecossistêmicos (ASE), assim como realizar uma breve revisão sistemática dos trabalhos indexados na plataforma Web of Science, associados a essa temática.

Diante da classificação apresentada pela Avaliação Ecossistêmica do Milênio (MEA, 2005), a polinização é um serviço ecossistêmico de regulação, imprescindível para a reprodução de espécies vegetais, contribuindo para a segurança alimentar e preservação da biodiversidade. Não obstante, a oferta deste serviço encontra-se ameaçada pelas constantes perdas de população de abelhas, silvestres e domesticadas, em decorrência das atividades agrícolas em todo mundo (MWEBAZE et al., 2018; SíLVIA et al., 2016). Conforme esta revisão, o número de publicações que avaliaram a polinização por abelhas aumentou consideravelmente nos últimos dez anos, representando $86,6 \%$ das publicações analisadas, sendo que os periódicos que mais publicaram foram: Agriculture, Ecosystems and Environment, Journal of Applied Ecology e Journal of Economic Entomology.

Dos benefícios identificados, $84,44 \%$ dos trabalhos associaram a polinização à produtividade de culturas agrícolas, enquanto $8,88 \%$ avaliaram a importância deste serviço ecossistêmico para a reprodução de plantas silvestres e 6,66\% para a agricultura urbana. Os trabalhos utilizaram métodos que avaliaram a produtividade das culturas analisadas tanto na presença quanto na ausência do serviço de polinização. Dessa forma, a produção de sementes por fruto foi um dos critérios diretamente associado ao seu tamanho e qualidade, fatores determinantes para a estipulação de valores de mercado.

Das 45 pesquisas analisadas, somente 07 realizaram a valoração econômica deste serviço utilizando diferentes métodos que captaram valores e escalas temporais e espaciais distintos. Os métodos Taxa de Dependência de Polinização (TDP), Custo de Reposição (CR), Análise de Rendimento Líquido (ARL) se baseiam nos valores de uso da polinização, enquanto a Disposição a Pagar (DAP) tem como princípio as preferências dos consumidores. Este último é o único método capaz de captar os valores de não uso ou intangíveis dos serviços ecossistêmicos, porém depende do conhecimento dos agentes econômicos acerca do bem que está sendo avaliado.

A partir daí, compreender a polinização por abelhas sob o olhar da ASE significa não reduzir os seus benefícios meramente a valores de mercado. Trata-se de uma proposta que aponta para a necessidade da construção de um novo paradigma que reorienta a relação entre homem e natureza. Deve-se reconhecer a limitações de estoque do capital natural e da sua capacidade de gerar fluxos ao longo do tempo, considerando o direito de escolha das futuras gerações. No caso da polinização por abelhas, a preservação destas dependerá de uma mudança nos moldes tradicionais das atividades agropecuárias que colocam em risco os habitats destes insetos.

Nesta revisão confirmou-se a hipótese de que a polinização por abelhas é de forma mais ampla reconhecida pelos seus benefícios para a agricultura. De acordo com a ASE, tais benefícios devem ser 
compreendidos sob uma proposta integradora que também leva em conta as dimensões sociais e ambientais relacionadas ao serviço ecossistêmico. Nesse caso, é importante investigar os desdobramentos destes benefícios para a produção de alimentos em quintais, para a agricultura familiar, para a agricultura urbana, para a reprodução de plantas silvestres e, consequentemente, para o equilíbrio dos ecossistemas. Pesquisas futuras poderão se concentrar nos benefícios para além dos valores de uso deste serviço ecossistêmico, contemplando, por exemplo, aspectos estéticos e culturais que podem ser obtidos a partir da manutenção de parques e jardins no interior de ambientes urbanos.

\section{REFERÊNCIAS}

AIZEN, M. A.; HARDER, L. D.. The global stock of domesticated honey bees is growing slower than agricultural demand for pollination. Current Biology, v.19, n.11, p.915918, 2009. DOI: http://doi.org/10.1016/j.cub.2009.03.071

ANDRADE, D. C.. Elementos facilitadores do entendimento das interfaces entre sistemas naturais e socioeconômicos. Holos, v.2, p.155, 2015. DOI: http://doi.org/10.15628/holos.2015.2125

ANDRADE, D. C.; ROMEIRO, A. R.. Valoração de serviços ecossistêmicos: por que e como avançar? Valoração de serviços ecossistêmicos: por que e como avançar?. Sustentabilidade em Debate, v.4, n.1, p.43-58, 2013. DOI: http://doi.org/10.18472/SustDeb.v4n1.2013.9199

ARTZ, D. R.; ALLAN, M. J.; WARDELL, G. I.; PITTS-SINGER, T. L.. Nesting site density and distribution affect Osmia lignaria (Hymenoptera: Megachilidae) reproductive success and almond yield in a commercial orchard. Insect Conservation and Diversity, v.6, n.6, p.715-724, 2013. DOI:

http://doi.org/10.1111/icad.12026

BALZAN, M. V.. Flowering banker plants for the delivery of multiple agroecosystem services. Arthropod-Plant Interactions, v.11, n.6, p.743-754, 2017. DOI: http://doi.org/10.1007/s11829-017-9544-2

BIRKIN, L.; GOULSON, D.. Using citizen science to monitor pollination services. Ecological Entomology, v.40, n.S1, p.311, 2015. DOI: http://doi.org/10.1111/een.12227

BLANCHE, K. R.; LUDWIG, J. A.; CUNNINGHAM, S. A.. Proximity to rainforest enhances pollination and fruit set in orchards. Journal of Applied Ecology, v.43, n.6, p.11821187, 2006. DOI: http://doi.org/10.1111/i.13652664.2006.01230.x

BPBES. Plataforma Brasileira de Biodiversidade e Serviços Ecossistêmicos. Relatório temático sobre polinização, polinizadores e produção de alimentos no Brasil. BPBES, 2019. DOI: http://doi.org/10.4322/978-85-60064-83-0

BRAAT, L. C.; GROOT, R.. The ecosystem services agenda:bridging the worlds of natural science and economics, conservation and development, and public and private policy. Ecosystem Services, v.1, n.1, p.4-15, 2012. DOI: http://doi.org/10.1016/j.ecoser.2012.07.011

BREEZE, T. D.; BAILEY, A. P.; BALCOMBE, K. G.; POTTS, S. G.. Pollination services in the UK: How important are honeybees?. Agriculture, Ecosystems and Environment, v.142, n.3-4, p.137-143, 2011. DOI: http://doi.org/10.1016/j.agee.2011.03.020

BRELAND, S.; TURLEY, N. E.; GIBBS, J.; ISAACS, R.; BRUDVIG, L. A.. Restoration increases bee abundance and richness but not pollination in remnant and post-agricultural woodlands. Ecosphere, v.9, n.9, 2018. DOI: http://doi.org/10.1002/ecs2.2435

BUCHHOLZ, S.; EGERER, M. H.. Functional ecology of wild bees in cities: towards a better understanding of traiturbanization relationships. Biodiversity and Conservation, v.29, n.9-10, p.2779-2801, 2020. DOI: http://doi.org/10.1007/s10531-020-02003-8

CARIVEAU, D. P.; WILLIAMS, N. M.; BENJAMIN, F. E.; WINFREE, R.. Response diversity to land use occurs but does not consistently stabilise ecosystem services provided by native pollinators. Ecology Letters, v.16, n.7, p.903-911, 2013. DOI: http://doi.org/10.1111/ele.12126

COSTA, C. C. A.; OLIVEIRA, F. L.. Polinização: serviços ecossistêmicos e o seu uso na agricultura. Pollination: ecosystem services and their use in agriculture. Revista Verde de Agroecologia e Desenvolvimento Sustentável, v.8, p.1-10, 2013.

CONSTANZA, R.; DALY, H. E.; BARTHOLOMEW, J. A.. Goals, agenda and policy recommendations for ecological economics. Environmental Accounting for Sustainable, p.120, 1991.

COSTANZA, R.; D'ARGE, R.; GROOT, R.; FARBRK, S.; GRASSO, M.; HANNON, B.; LIMBURG, K.; NAEEM S.; O'NEILL, R. V.; PARUELO, J.; RASKIN, R. G.; SUTTONKK, P.; DEN BELT, M.. The value of the world's ecosystem services and natural capital. Nature, v.387, n.6630, p.253-260, 1997. DOI: http://doi.org/10.1038/387253a0

COSTANZA, R.; GROOT, R.; BRAAT, L.; KUBISZEWSKI, I.; FIORAMONTI, L.; SUTTON, P.; FARBER, S.; GRASSO, M.. Twenty years of ecosystem services: How far have we come and how far do we still need to go? Ecosystem Services, v.28, p.1-16, 2017. DOI: http://doi.org/10.1016/j.ecoser.2017.09.008

COSTANZA, R.. Valuing natural capital and ecosystem services toward the goals of efficiency, fairness, and sustainability. Ecosystem Services, v.43, p.101096, 2020. DOI: http://doi.org/10.1016/i.ecoser.2020.101096 
DAHAL, R. P.; GRALA, R. K.; GORDON, J. S.; PETROLIA, D. R.; MUNN, I. A.. Estimating the willingness to pay to preserve waterfront open spaces using contingent valuation. Land Use Policy, v.78, p.614-626, 2018. DOI: http://doi.org/10.1016/j.landusepol.2018.07.027

DAVIS, A. Y.; LONSDORF, E. V.; SHIERK, C. R.; MATTESON, K. C.; TAYLOR, J. R.; LOVELL, S. T.; MINOR, E. S.. Enhancing pollination supply in an urban ecosystem through landscape modifications. Landscape and Urban Planning, v.162, p.157166, 2017. DOI:

http://doi.org/10.1016/j.landurbplan.2017.02.011

EHRLICH R. P.; MOONEY A. H.. Extinction, substitution and ecosystem services. BioScience, v.33, n.4, p.248-254, 2013. DOI: http://doi.org/10.2307/1309037

ELIAS, M. A. S.; BORGES, F. J. A.; BERGAMINI, L. L.; FRANCESCHINELLI, E. V.; SUJII, E. R.. Climate change threatens pollination services in tomato crops in Brazil. Agriculture, Ecosystems and Environment, v.239, p.257264, 2017. DOI: http://doi.org/10.1016/i.agee.2017.01.026

FARLEY, J.. The role of prices in conserving critical natural capital. Conservation Biology, v.22, n.6, p.1399-1408, 2008. DOI: http://doi.org/10.1111/j.1523-1739.2008.01090.x

GAGLIANONE, M. C.; CAMPOS, M. J. O.; FRANCESCHINELLI, E.; DEPRÁ, M. S.; SILVA, P. N.; MONTAGNANA, P. C.; HAUTEQUESTT, A. P.; MORAES, M. C. M.; CAMPOS, L. A. O.. Plano de manejo para os polinizadores do tomateiro. 2015.

GALBRAITH, S. M.; VIERLING, L. A.; BOSQUE-PÉREZ, N. A.. Remote sensing and ecosystem services: Current status and future opportunities for the study of bees and pollinationrelated services. Current Forestry Reports, v.1, n.4, p.261274, 2015. DOI: http://doi.org/10.1007/s40725-015-0024-6

GALLAI, N.; SALLES, J. M.; SETTELE, J.; VAISSIÈRE, B. E.. Economic valuation of the vulnerability of world agriculture confronted with pollinator decline. Ecological Economics, v.68, n.3, p.810-821, 2008. DOI:

http://doi.org/10.1016/j.ecolecon.2008.06.014

GARIBALDI, L. A.; CARVALHEIRO, L. G.; VAISSIÈRE, B. E.; GEMMILL-HERREN, B.; HIPÓLITO, J.; FREITAS, B. M.; NGO, H. T.; AZZU, N.; SÁEZ, A.; ASTRÖM, J.; AN, J.; BLOCHTEIN, B.; BUCHORI, D.; GARCÍA, F. J. C.; SILVA, F. O.; DEVKOTA, K.; RIBEIRO, M. F.; FREITAS, L.; GAGLIANONE, M. C.; GOSS M.; IRSHAD, M.; KASINA, M.; FILHO, A. J. S. P.; KIILL, L. H. P.; KWAPONG, P.; PARRA, G. N.; PIRES, C.; PIRES, V.; RAWAL, R. S.; RIZALI, A.; SARAIVA, A. M.; VELDTMAN, R.; VIANA, B. F.; WITTER, S.; ZHANG, H.. Mutually beneficial pollinator diversity and crop yield outcomes in small and large farms. Science, v.351, n.6271, p.388-391, 2016. DOI: http://doi.org/10.1126/science.aac7287

GIANNINI, T. C.; BOFF, S.; CORDEIRO, G. D.; CARTOLANO, E. A.; VEIGA, A. K.; IMPERATRIZ-FONSECA, V. L.; SARAIVA, A. $M$.. Crop pollinators in Brazil: a review of reported interactions. Apidologie, v.46, n.2, p.209-223, 2015a. DOI: http://doi.org/10.1007/s13592-014-0316-z

GIANNINI, T. C.; CORDEIRO, G. D.; FREITAS, B. M.; SARAIVA, A. M.; IMPERATRIZ-FONSECA, V. L.. The dependence of crops for pollinators and the economic value of pollination in
Brazil. Journal of Economic Entomology, v.108, n.3, p.849857, 2015b. DOI: http://doi.org/10.1093/jee/tov093

GÓMEZ-BAGGETHUN, E.; GROOT, R.; LOMAS, P. L.; MONTES, C.. The history of ecosystem services in economic theory and practice: From early notions to markets and payment schemes. Ecological Economics, v.69, n.6, p.1209-1218, 2010. DOI: http://doi.org/10.1016/i.ecolecon.2009.11.007

GOULD, R. K.;RICKETTS, T. H.; HOWARTH, R. B.; TELLE, S.; GLADKIKH, T.; POSNER, S.; GOUREVITCH, J.; YOSHIDA, Y.. How ecosystem services research can advance ecological economics principles. In: COSTANZA, R.. How ecosystem services research can advance ecological economics principles. Sustainable Wellbeing Futures, p.127-150, 2020. DOI: http://doi.org/10.4337/9781789900958.00017

HIPÓLITO, J.; SOUSA, B. S. B.; BORGES, R. C.; BRITO, R. M.; JAFFÉ, R.; DIAS, S.; IMPERATRIZ-FONSECA, V. L.; GIANNINI, T. C.. Valuing nature's contribution to people: The pollination services provided by two protected areas in Brazil. Global Ecology and Conservation, v.20, 2019. DOI: http://doi.org/10.1016/j.gecco.2019.e00782

HOSHIDE, A. K.; DRUMMOND, F. A.; STEVENS, T. H.; VENTURINI, E. M.; HANES, S. P.; SYLVIA, M. M.; LOFTIN, C. S.; YARBOROUGH, D. E.; AVERILL, A. L.. What is the value of wild bee pollination for wild blueberries and cranberries, and who values it? Environments - MDPI, v.5, n.9, p.1-24, 2018. DOI: http://doi.org/10.3390/environments5090098

IMPERATRIZ-FONSECA, V. L.; NUNES-SILVA, P.. As abelhas, os serviços ecossistêmicos e o Código Florestal Brasileiro. Biota Neotropica, v.10, n.4, p.59-62, 2010. DOI: http://doi.org/10.1590/s1676-06032010000400008

ISAACS, R.; KIRK, A. K.. Pollination services provided to small and large highbush blueberry fields by wild and managed bees. Journal of Applied Ecology, v.47, n.4, p.841-849, 2010. DOI: http://doi.org/10.1111/j.1365-2664.2010.01823.x

KASINA, J. M.; MBURU, J.; KRAEMER, M.; HOLM-MUELLER, K.. Economic benefit of crop pollination by bees: A case of kakamega small-holder farming in Western Kenya. Journal of Economic Entomology, v.102, n.2, p.467-473, 2009. DOI: http://doi.org/10.1603/029.102.0201

KLATT, B. K.; HOLZSCHUH, A.; WESTPHAL, C.; CLOUGH, Y.; SMIT, I.; PAWELZIK, E.; TSCHARNTKE, T.. Bee pollination improves crop quality, shelf life and commercial value. Proceedings of the Royal Society B: Biological Sciences, v.281, n.1775, 2013. DOI: http://doi.org/10.1098/rspb.2013.2440

KLEIN, A.; VAISSIE, B. E.; CANE, J. H.; STEFFAN-DEWENTER, I.; CUNNINGHAM, S. A.; KREMEN, C.; TSCHARNTKE, T.. Importance of pollinators in changing landscapes for world crops. Proceedings of the Royal Society B, v.274, p.303-313, 2007. DOI: http://doi.org/10.1098/rspb.2006.3721

LIU, X. W.; CHESTERS, D.; DAI, Q. Y.; NIU, Z. Q.; BECKSCHÄFER, P.; MARTIN, K.; ZHU, C. D.. Integrative Profiling of Bee Communities from Habitats of Tropical Southern Yunnan (China). Scientific Reports, v.7, n.1, p.1-14, 2017. DOI: http://doi.org/10.1038/s41598-017-05262-8

MALLINGER, R. E.; GRATTON, C.. Species richness of wild 
bees, but not the use of managed honeybees, increases fruit set of a pollinator-dependent crop. Journal of Applied

Ecology, v.52, n.2, p.323-330, 2015. DOI:

http://doi.org/10.1111/1365-2664.12377

MARQUES. L.. Capitalism and environmental collapse. Campinas: Springer, 2020.

MARTINS, K. T.; GONZALEZ, A.; LECHOWICZ, M. J.. Pollination services are mediated by bee functional diversity and landscape context. Agriculture, Ecosystems and Environment, v.200, p.12-20, 2015. DOI: http://doi.org/10.1016/j.agee.2014.10.018

MEA. Millenium Ecossystm Assessment. Ecosystems and Human Well-being: Synthesis. Washington: Island Press, 2005.

MSU. Michigan State University. Feeding the world in $\mathbf{2 0 5 0}$ and beyond - Part 1: Productivity challenges. Michigan State University Extension, 2018.

MOHER, D.; LIBERATI, A.; TETZLAFF, J.; ALTMAN, D. G.; GROUP, The Prisma. Preferred Reporting Items for Systematic Reviews and Meta-Analyses: The PRISMA Statement. Plos Med. v.6, n.7, 2009. DOI: http://doi.org/10.1371/journal.pmed.1000097

MWEBAZE, P.; MARRIS, G. C.; BROWN, M.; MACLEOD, A.; JONES, G.; BUDGE, G. E.. Measuring public perception and preferences for ecosystem services: A case study of bee pollination in the UK. Land Use Policy, v.71, p.355-362, 2018. DOI: http://doi.org/10.1016/j.landusepol.2017.11.045

NASCIMENTO, W. M.; GOMES, E. M. L.; BATISTA, E. A.; FREITAS, R. A.. Utilização de agentes polinizadores na produção de sementes de cenoura e pimenta doce em cultivo protegido. Horticultura Brasileira, v.30, n.3, p.494498, 2012. DOI: http://doi.org/10.1590/S0102$\underline{05362012000300023}$

NICHOLLS, E.; ELY, A.; BIRKIN, L.; BASU, P.; GOULSON, D.. The contribution of small-scale food production in urban areas to the sustainable development goals: a review and case study. Sustainability Science, v.15, n.6, p.1585-1599, 2020. DOI: http://doi.org/10.1007/s11625-020-00792-z

OLLERTON, J.; WINFREE, R.; TARRANT, S.. How many flowering plants are pollinated by animals?. Oikos, v.120, n.3, p.321-326, 2011. DOI: http://doi.org/10.1111/i.1600$\underline{0706.2010 .18644 . x}$

PFISTER, S. C.; ECKERTER, P. W.; SCHIRMEL, J.; CRESSWELL, J. E.; ENTLING, M. H.. Sensitivity of commercial pumpkin yield to potential decline among different groups of pollinating bees. Royal Society Open Science, v.4, n.5, 2017. DOI: http://doi.org/10.1098/rsos.170102

POPAK, A. E.; MARKWITH, S. H.; STRANGE, J.. Economic Valuation of Bee Pollination Services for Passion Fruit (Malpighiales: Passifloraceae) Cultivation on Smallholding Farms in São Paulo, Brazil, Using the Avoided Cost Method.
Journal of Economic Entomology, v. 112, n.5, p.2049-2054, 2019. DOI: http://doi.org/10.1093/iee/toz169

PORTO, R. G.; ALMEIDA, R. F.; CRUZ-NETO, O.; TABARELLI, M.; VIANA, B. F.; PERES, C. A.; LOPES, A. V.. Pollination ecosystem services: A comprehensive review of economic values, research funding and policy actions. Food Security, v.12, p.1425-1442, 2020. DOI: http://doi.org/10.1007/s12571-020-01043-w

ROGERS, S. R.; TARPY, D. R.; BURRACK, H. J.. Bee species diversity enhances productivity and stability in a perennial crop. Plos One, v.9, n.5, 2014. DOI: http://doi.org/10.1371/journal.pone.0097307

RUNDLÖF, M.; LUNDIN, O.; BOMMARCO, R.. Annual flower strips support pollinators and potentially enhance red clover seed yield. Ecology and Evolution, v.8, n.16, p.7974-7985, 2018. DOI: http://doi.org/10.1002/ece3.4330

SÍLVIA, C.; PIRES, S.; PEREIRA, F. M.; TERESA, M..

Enfraquecimento e perda de colônias de abelhas no Brasil : há casos de CCD?. Pesq. Agropec. Bras., v.51, n.5, p.422442, 2016. DOI: http://doi.org/10.1590/S0100204X2016000500003

SUTTER, L.; HERZOG, F.; DIETEMANN, V.; CHARRIÈRE, J.; ALBRECHT, M.. Par Les Insectes Dans L' Agriculture Suisse. Recherche Agronomique Suisse, v.8, n.9, p.332-339, 2017.

UN. United Nations. $68 \%$ of the world population projected to live in urban areas by 2050, says UN. Department of Economic and Social Affairs. New York: UN, 2018.

VIEIRA, P. F. S. P.; CRUZ, D. O.; GOMES, M. F. M.; L. A. O.; LIMA, J.. Valor econômico da polinização por abelhas mamangavas no cultivo do maracujá-amarelo. Revibec: Revista Ibero-Americana de Economía Ecológica, v.15, p.4353, 2010.

WINFREE, R.; WILLIAMS, N. M.; DUSHOFF, J.; KREMEN, C.. Native bees provide insurance against ongoing honey bee losses. Ecology Letters, v.10, n.11, p.1105-1113, 2007. DOI: http://doi.org/10.1111/j.1461-0248.2007.01110.x

WINFREE, R.; GROSS, B. J.; KREMEN, C.. Valuing pollination services to agriculture. Ecological Economics, v.71, n.1, p.8088, 2011. DOI:

http://doi.org/10.1016/i.ecolecon.2011.08.001

WOODCOCK, B. A.; EDWARDS, M.; REDHEAD, J.; MEEK, W. R.; NUTTALL, P.; FALK, S.; NOWAKOWSKI, M.; PYWELL, R. F.. Crop flower visitation by honeybees, bumblebees and solitary bees: Behavioural differences and diversity responses to landscape. Agriculture, Ecosystems and Environment, v.171, p.1-8, 2013. DOI: http://doi.org/10.1016/i.agee.2013.03.005

YOUNG, A. M.; GÓMEZ-RUIZ, P. A.; PEÑA, J. A.; UNO, H.; JAFFE, R.. Wind speed affects pollination success in blackberries. Sociobiology, v.65, n.2, p.225-231, 2018. DOI: http://doi.org/10.13102/sociobiology.v65i2.1620

A CBPC - Companhia Brasileira de Produção Científica (CNPJ: 11.221.422/0001-03) detém os direitos materiais desta publicação. Os direitos referem-se à publicação do trabalho em qualquer parte do mundo, incluindo os direitos às renovações, expansões e disseminações da contribuição, bem como outros direitos subsidiários. Todos os trabalhos publicados eletronicamente poderão posteriormente ser publicados em coletâneas impressas sob coordenação da Sustenere Publishing, da Companhia Brasileira de Produção Científica e seus parceiros autorizados. Os (as) autores (as) preservam os direitos autorais, mas não têm permissão para a publicação da contribuição em outro meio, impresso ou digital, em português ou em tradução. 